\title{
Development, Implementation, and
} Patient Perspectives of the Women's Integrative Sexual Health Program: A Program Designed to Address the Sexual Side Effects of Cancer Treatment

\author{
LORI A. SEABORNE, MPAS, PA-C, MEGAN PETERSON, DNP, DAVID M. KUSHNER, MD, \\ JANELLE SOBECKI, MD, MA, and JOANNE K. RASH, MPAS, PA-C
}

From University of Wisconsin Carbone Cancer Center, Madison, Wisconsin

Authors' disclosures of conflicts of interest are found at the end of this article.

Correspondence to: Lori A. Seaborne, MPAS, PA-C, University of Wisconsin Hospital and Clinics, 600 Highland Ave G3/127, Madison, WI 53792. E-mail: seaborne@surgery.wisc.edu

https://doi.org/10.6004/jadpro.2021.12.1.3 (c) 2021 Harborside $^{\text {TM }}$

\section{Abstract}

Background: Advanced practitioners (APs) are a growing demographic in survivorship care. One goal of survivorship care is to manage consequences of cancer treatments. Sexual dysfunction from prior therapies can impact quality of life. Advanced practitioners are perfectly poised to provide care for sexual problems. This article will describe the development and implementation of the Women's Integrative Sexual Health (WISH) program by APs within a comprehensive cancer center and describe patient perspectives of care provided. Methods: Two physician assistants working in gynecologic oncology at the University of Wisconsin Carbone Cancer Center implemented a program to address sexual side effects of cancer treatment. An online survey was sent out to all patients seen in the WISH program since inception. Results: Between November 2013 and July 2019, 228 patients were seen in the WISH program. A total of 113 women responded (median age: 53 years, range: 31-77; 68\% postmenopausal; response rate: $53.8 \%$ ). Most had breast (57\%) or gynecologic (32\%) cancers. When asked how helpful the WISH program was, $88 \%$ reported that it was at least somewhat helpful. Almost all (95\%) reported they would recommend the WISH program to other women. Conclusion: The WISH program enhances comprehensive survivorship care of female cancer survivors. Women report they benefit from care for sexual issues after cancer treatments. Advanced practitioners working in oncology are uniquely positioned to educate themselves, take leadership roles in the development and implementation of programs, and provide care to women affected by sexual side effects after cancer. 
dvanced practitioners (APs) are an increasingly valuable part of the care team in oncology settings. They are being utilized to provide a wide variety of services, including managing acute outpatient issues, prescribing chemotherapy, rounding on inpatients, and assisting in surgery (Corcoran, Dunne, \& McCabe, 2015; Shlulman, 2013; Rash, Lyle, Jairam-Thodla, \& Ioffe, 2018). In one observational study following women with breast cancer over a 4-year time period after diagnosis, the number of billable visits to APs increased from $36 \%$ to $61 \%$ (Yen, Laud, McGinley, Pezzin, \& Nattinger, 2019). This indicates that APs are a growing demographic on survivorship care teams.

As of January 1, 2019, there were 16.9 million cancer survivors in the United States, 8.8 million of whom were women (Miller et al., 2019). One of the goals of survivorship care is to manage consequences of cancer and its treatment (Morgan \& Tarbi, 2016). Sexual dysfunction from prior therapies (surgery, chemotherapy, radiation therapy, endocrine therapy) impacts cancer survivors' quality of life (Huffman, Hartenback, Carter, Rash, \& Kushner, 2016). It has been documented that most of the cancers that affect women affect their sexual organs, and therefore sexual function (Lindau, Abramsohn, \& Matthews, 2015).

Sexual health is important to women after cancer treatment. In one survey of breast and gynecologic cancer survivors, over $40 \%$ of women stated they were interested in receiving care for sexual side effects, but only $7 \%$ sought help for those issues (Hill et al., 2011). Communication about sexual health concerns between oncology patients and their providers does not occur routinely (Flynn et al., 2012). In order to offer comprehensive care to female cancer survivors, sexual side effects deserve evaluation. Advanced practitioners are perfectly poised to provide this care.

The aim of this article is to describe the development and implementation of a comprehensive sexual health program for women with cancer implemented by APs within a comprehensive cancer center and evaluate patient care experiences in this clinic.

\section{METHODS}

\section{Development of the WISH Program}

Two physician assistants (PAs) working in gynecologic oncology at the University of Wisconsin (UW) Hospital identified a need for a comprehensive sexual health program after years of fielding the sexual health questions and concerns of their patients. Women reported multiple issues, including loss of sexual interest, lack of ability to reach orgasm, and sexual pain. Leadership in the Division of Gynecologic Oncology, the Department of Obstetrics and Gynecology, and the UW Carbone Cancer Center were approached to discuss how best to meet the sexual health needs of all women affected by cancer at the institution. Leadership was supportive of dedicated AP time and provided monetary support for supplies and marketing. A gynecologic oncologist played a supportive and administrative role as the medical director of the program without providing direct patient care.

The Program for Integrative Sexual Medicine (PRISM) team at the University of Chicago provided mentorship on program development. The Women's Integrative Sexual Health (WISH) team collaborated with the PRISM team to participate in a shared, prospective patient registry, which collects demographic and clinical information as well as sexual health outcomes with a goal of expanded data generation in this patient population. Each patient seen in the WISH program is offered the option of joining the patient registry.

Templates for appropriate medical record documentation were developed in conjunction with the institution's IT department. The decision was made to create a unique department within the electronic health record (EHR) in order to allow formal referrals to be made by oncology providers at the institution. This protects patient privacy by clearly stating in the EHR that a visit was done within the WISH department.

The hospital's public relations team assisted in the design of the WISH program brochure and website page. Newsletters were published to advertise the new service. The WISH team PAs presented to the providers, nurses, and medical assistants within multiple disease-site programs at the cancer center to raise awareness of the new program. Outreach efforts included presentations at the Carbone Cancer Center's grand rounds and 
annual conference, as well as a local chapter of Gilda's Club. The UW department of Obstetrics and Gynecology highlighted the program in a blog and podcast.

\section{Advanced Practitioner Skill Development and Interdisciplinary Team Building}

One of the early barriers that needed to be addressed was the lack of sexual health education offered to medical providers during training. A 2014 survey of United States PA programs revealed that of the 106 responding programs, only $40 \%$ covered diagnosis and management of female sexual function problems (Seaborne, Prince, $\&$ Kushner, 2015). Therefore, it was a top priority to seek out accurate and up-to-date information about managing sexual health concerns in women. In order to improve their skills performing comprehensive histories and physical exams and managing sexual concerns, the team read textbooks, journal articles, and reputable lay publications. They also attended professional conferences and collaborated with existing sexual health programs and local sexual health experts. One of the PAs completed a formal sexual health education program and became a certified sexuality counselor through the American Association of Sexuality Educators, Counselors and Therapists (AASECT).

Creating a network of professionals in the community was also essential. The team partnered with UW pelvic floor physical therapists, who shared their wealth of knowledge regarding assessment and management of pelvic floor disorders, as well as health psychologists, gynecologists, relationship counselors, and AASECTcertified sex therapists (Table 1). Having an appropriate referral base allowed for comprehensive care of sexual health changes after cancer.

The AP team meets with the WISH medical director every other month to discuss pertinent logistical issues. There are also quarterly meetings with the UW pelvic floor physical therapists to discuss mutual patients. Regular meetings keep the

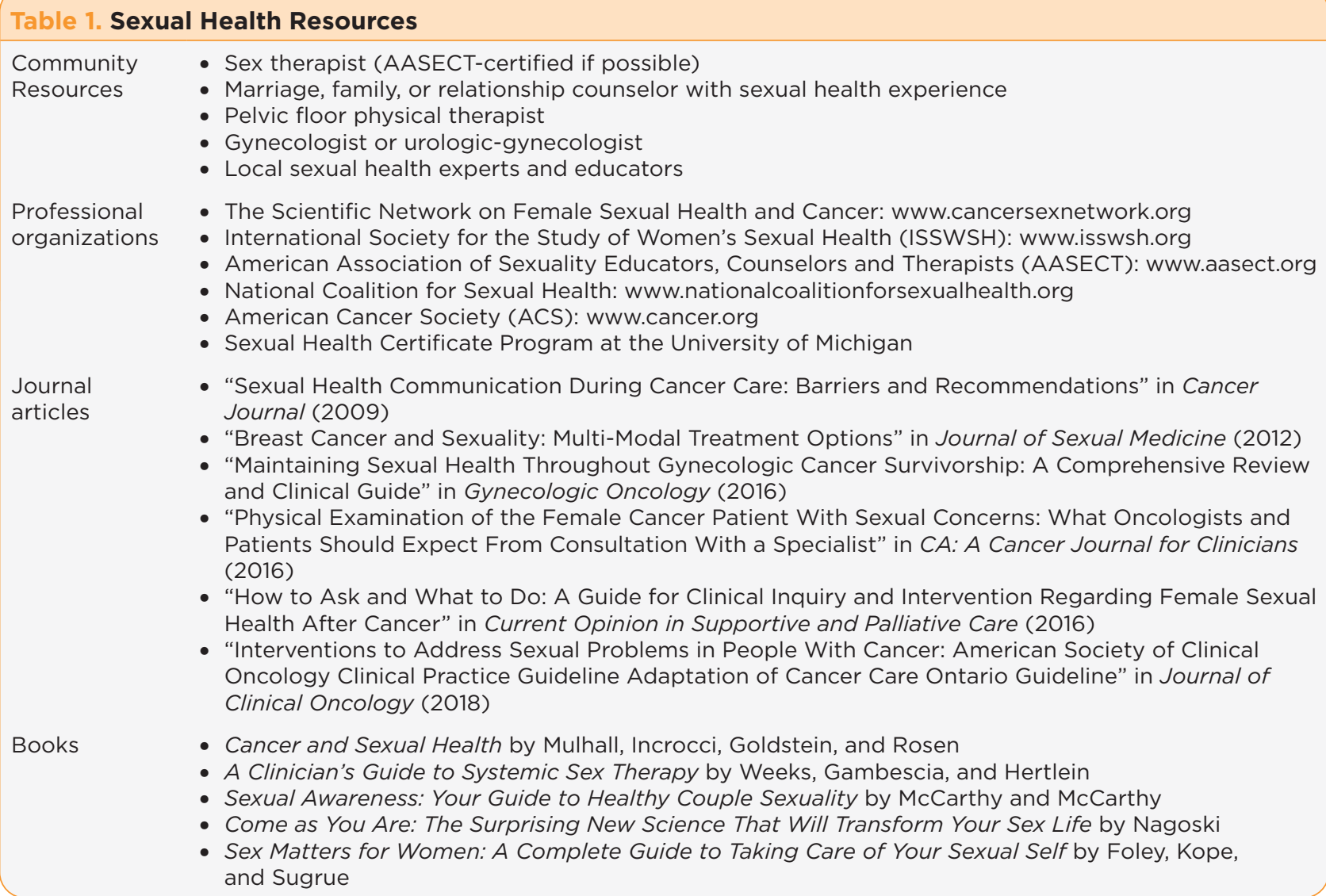

- "Breast Cancer and Sexuality: Multi-Modal Treatment Options" in Journal of Sexual Medicine (2012)

- "Maintaining Sexual Health Throughout Gynecologic Cancer Survivorship: A Comprehensive Review and Clinical Guide" in Gynecologic Oncology (2016)

- "Physical Examination of the Female Cancer Patient With Sexual Concerns: What Oncologists and Patients Should Expect From Consultation With a Specialist" in CA: A Cancer Journal for Clinicians (2016)

- "How to Ask and What to Do: A Guide for Clinical Inquiry and Intervention Regarding Female Sexual Health After Cancer" in Current Opinion in Supportive and Palliative Care (2016)

- "Interventions to Address Sexual Problems in People With Cancer: American Society of Clinical Oncology Clinical Practice Guideline Adaptation of Cancer Care Ontario Guideline" in Journal of Clinical Oncology (2018)

Books - Cancer and Sexual Health by Mulhall, Incrocci, Goldstein, and Rosen

- A Clinician's Guide to Systemic Sex Therapy by Weeks, Gambescia, and Hertlein

- Sexual Awareness: Your Guide to Healthy Couple Sexuality by McCarthy and McCarthy

- Come as You Are: The Surprising New Science That Will Transform Your Sex Life by Nagoski

- Sex Matters for Women: A Complete Guide to Taking Care of Your Sexual Self by Foley, Kope, and Sugrue 
team working toward institutional and programmatic goals and allow for ongoing team building.

For APs interested in developing their skills in this area, multiple journal articles have been published offering guidelines regarding the importance of good communication, as well as how to complete a physical examination and manage sexual symptoms in cancer survivors (Bober et al., 2016; Carter et al., 2018; Huffman et al., 2016; Krychman \& Katz, 2012; Lindau et al., 2016; Park, Norris, \& Bober, 2009).

\section{Implementation of the WISH Program}

The team adopted the two-visit model used by the PRISM program for each new patient, which allows adequate time for history taking, physical exam, and counseling. The initial 60-minute visit includes a sexual health history, assessment of current and past sexual relationships, a review of systems, and a detailed discussion of current sexual concerns. The AP determines diagnoses and creates a tailored plan that includes in-person education, written materials, and medical intervention. The second visit includes follow-up discussion, physical exam (including a detailed pelvic exam), supplemental education, and additional customization of the treatment plan. Appropriate referrals to outside services are also performed after physical exam is completed. Subsequent visits are scheduled as needed to monitor progress and provide further recommendations.

Initially, the two PAs saw patients together to allow for collaboration, team learning, and reviewing consent for the patient registry. As demand increased, they started seeing patients individually. One of the PAs currently works with breast cancer survivors, and remains active in the WISH program. A nurse practitioner in gynecologic oncology was added to the team to further improve access for patients.

\section{Patient Survey Methods}

The project was determined to be a quality improvement project by the IRB and was therefore IRB exempt. A survey was designed to ascertain patient care experiences in the WISH program. This survey included questions regarding sexual concerns and whether those concerns improved after a visit to the program (Appendix A).
The survey was sent electronically to all patients who were seen in the WISH program from inception in November 2013 to July 2019. Participants were identified by internal clinic records. No patients were excluded. All patients received an e-mail 3 days prior to survey administration introducing the survey and providing instructions. Each participant received two e-mail reminders over a 2 -week period and responses were collected for a total of 6 weeks.

The survey was sent to 228 patients, and email delivery failure notices were received from 18 individuals. Therefore, a total of 210 women received the survey.

\section{RESULTS}

A total of 113 women (median age: 51 years, range: $28-76$; 68\% postmenopausal) responded (response rate: $53.8 \%$ ). Most had breast (57\%) or gynecologic (32\%) cancers. First visits spanned the entire 5 years of operation (Table 2). Women were asked to identify the sexual complaint(s) that were the impetus for their visit to the WISH program, as well as their perspective on the care they received.

The women who responded reported concerns in all areas of sexual functioning: sexual

\begin{tabular}{|c|c|c|c|}
\hline Demographic & Value & Number & Percentage \\
\hline Age & $\begin{array}{l}30 \text { and under } \\
31-40 \\
41-50 \\
51-60 \\
61-70 \\
\text { Over } 70 \\
\text { NA }\end{array}$ & $\begin{array}{l}5 \\
17 \\
34 \\
29 \\
20 \\
4 \\
4\end{array}$ & $\begin{array}{l}4 \\
15 \\
30 \\
26 \\
18 \\
4 \\
4\end{array}$ \\
\hline Cancer type ${ }^{a}$ & $\begin{array}{l}\text { Breast } \\
\text { Gynecologic } \\
\text { Hematologic } \\
\text { Gastrointestinal }\end{array}$ & $\begin{array}{l}64 \\
37 \\
9 \\
9\end{array}$ & $\begin{array}{l}57 \\
32 \\
8 \\
8\end{array}$ \\
\hline $\begin{array}{l}\text { Years since } \\
\text { first visit }\end{array}$ & $\begin{array}{l}0 \\
1 \\
2 \\
3 \\
4 \\
5\end{array}$ & $\begin{array}{l}6 \\
23 \\
12 \\
12 \\
6 \\
12\end{array}$ & $\begin{array}{l}8 \\
32 \\
16 \\
16 \\
8 \\
16\end{array}$ \\
\hline \multicolumn{4}{|c|}{$\begin{array}{l}\text { Note. Gynecologic = uterine, ovarian, cervical, vulvar, } \\
\text { vaginal, fallopian tube; hematologic = leukemia, } \\
\text { lymphoma, multiple myeloma, Hodgkin lymphoma; } \\
\text { gastrointestinal = rectal, anal, colon, gastric } \\
\text { aAdds up to more than } 100 \% \text { due to several women } \\
\text { reporting more than one cancer. }\end{array}$} \\
\hline
\end{tabular}




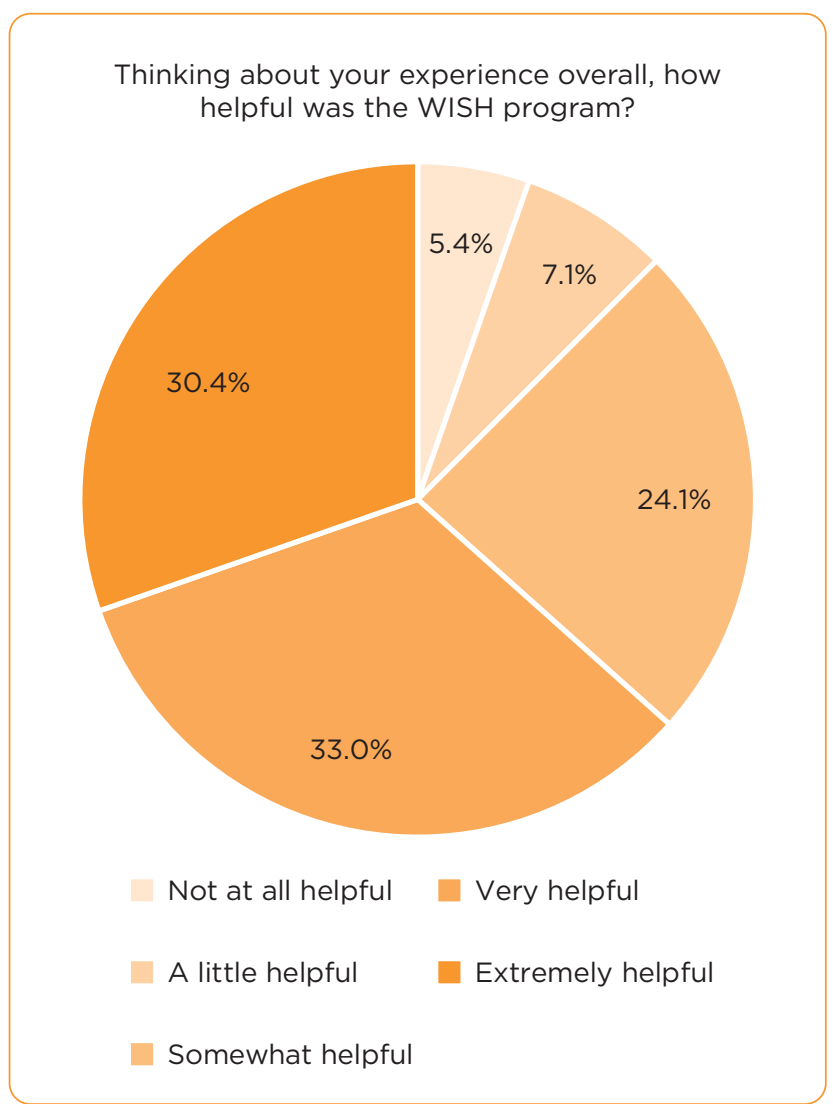

Figure 1. Helpfulness of the WISH program.

pain (88\%), vaginal dryness (86\%), decreased libido (84\%), orgasm concerns (58\%), and communication issues with the partner (47\%). Women were then asked if they noticed improvements in those same areas. Most women reported improvement in all areas, and most of those women who reported improvement also reported that the improvement persisted over time (Table 3 ).

When women were asked if they believed they had a good general understanding of the issues related to sexual changes after cancer, 62 women (55\%) stated that they did. However, 104 women (92\%) replied that they had improvement in their understanding after their visit to the WISH program, indicating that the counseling was effective in educating women about their concerns.

When asked how helpful their experience in the WISH program was, 99 of 113 (88\%) reported that it was at least somewhat helpful (Figure 1). Seventy-five out of 110 (68\%) said their experience in the WISH program improved their overall sexual function. Eighty out of 112 (71\%) thought their sexual function would be worse if they had not been seen in the WISH program (Figure 2). Almost all (95\%) reported they would recommend the WISH program to other women (Figure 3).

\section{CONCLUSION}

Advanced practitioners played a central leadership role in the successful development and implementation of the WISH program. WISH is now staffed by two PAs and one nurse practitioner who specialize in the care of gynecologic and breast cancer survivors. Patient evaluation in the WISH program is an adjunct to the usual practice, and is available to women with any type of cancer. The WISH program adds to the comprehensive nature of survivorship care for women after cancer treatment at the UW Carbone Cancer Center. Women report that they benefit from this survivorship service. Advanced practitioners working in oncology are uniquely positioned to educate themselves, take leadership roles in the development and implementation of programs, and provide care to women affected by sexual side effects after cancer.

\section{Acknowledgment}

The authors gratefully acknowledge the financial support of the University of Wisconsin Foundation through the John and Jeanne Flesch Professorship Fund in Gynecologic Oncology.

\section{Table 3. Reported Sexual Function Concerns and Improvement}

\begin{tabular}{|llll|}
\hline Sexual function concern & $\begin{array}{l}\text { Reported concerns, } \\
\text { no. (\%) }\end{array}$ & $\begin{array}{l}\text { Reported improvement, } \\
\text { no. (\%) }\end{array}$ & $\begin{array}{l}\text { Improvement persisted over } \\
\text { time, no. (\%) }\end{array}$ \\
Communication & $52(47 \%)$ & $39(75 \%)$ & $30(77 \%)$ \\
Vaginal dryness & $96(86 \%)$ & $69(73 \%)$ & $56(81 \%)$ \\
Pain with penetration & $99(88 \%)$ & $56(58 \%)$ & $39(70 \%)$ \\
Libido concerns & $94(84 \%)$ & $54(57 \%)$ & $39(72 \%)$ \\
Orgasm concerns & $65(58 \%)$ & $37(58 \%)$ & $26(70 \%)$ \\
\hline
\end{tabular}


After your experience with the WISH program, do you think your sexual functioning would be worse if you had not visited the WISH program?

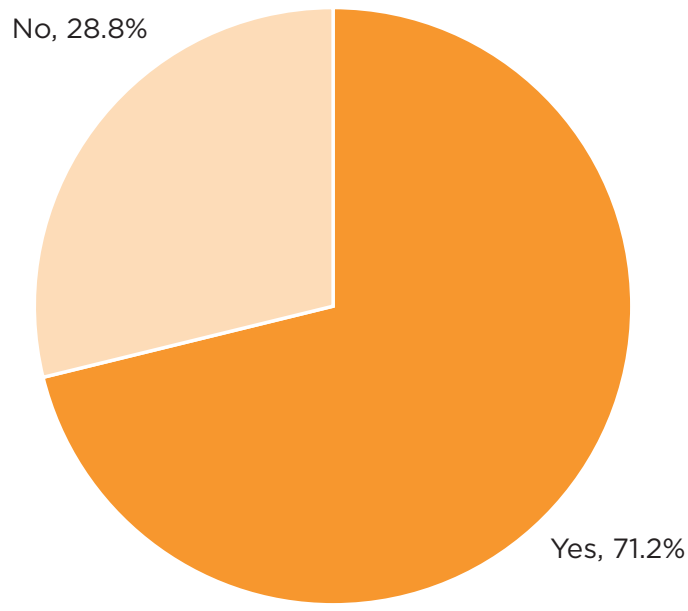

Figure 2. Sexual function following WISH program intervention.

The authors would like to express their appreciation to Stacy Tessler Lindau MD, MAPP, and Emily Abrahamson, MPH, and their team at the Program for Integrative Sexual Medicine (PRISM) at the University of Chicago for their support and mentorship during the development of the WISH program.

The authors would like to thank Ellen Barnard, MSSW, CSE, at A Woman's Touch Sexuality Resource Center, for her endless knowledge and ongoing support; Sarah Duffy, MPT, for sharing her knowledge of the pelvic floor; and Ahmed AlNiaimi, MD, for sharing his expertise regarding quality improvement research.

\section{Disclosure}

The authors have no conflicts of interest to disclose.

\section{References}

Bober, S. L., Reese, J. B., Barbera, L., Bradford, A., Carpenter, K., Goldfarb, S.,...Carter, J. (2016). How to ask and what to do: A guide for clinical inquiry and intervention regarding female sexual health after cancer. Current Opinion in Supportive and Palliative Care, 10(1), 44-54. https://doi. org/10.1097/SPC.0000000000000186

Carter, J., Lacchetti, C., Andersen, B. L., Barton, D. L., Bolte, S., Damast, S.,...Rowland, J. H. (2018). Interventions to address sexual problems in people with cancer: American Society of Clinical Oncology Clinical Practice Guide-
After your experience with the WISH program would you recommend it to other women with sexual health problems after cancer?

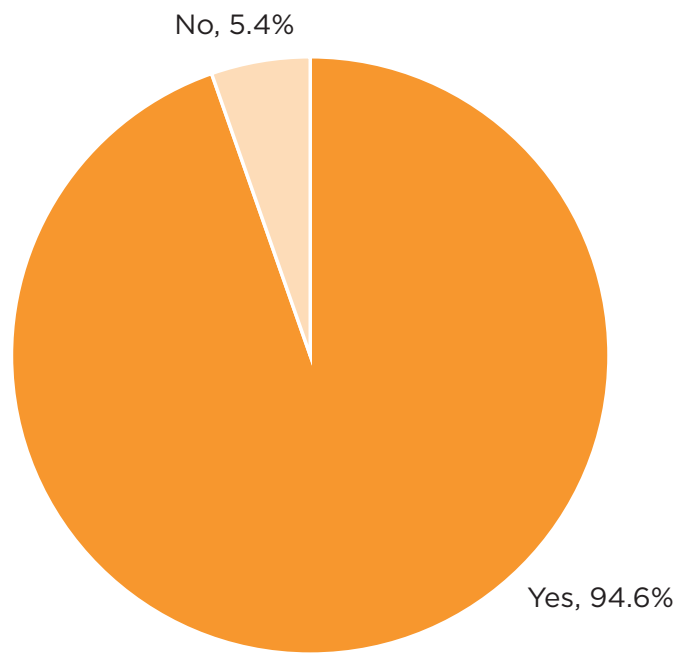

Figure 3. WISH program patient recommendations.

line Adaptation of Cancer Care Ontario Guideline. Journal of Clinical Oncology, 36(5), 492-511. https://doi. org/10.1200/JCO.2017.75.8995

Corcoran, S., Dunne, M., \& McCabe, M. S. (2015). the role of advanced practice nurses in cancer survivorship care. Seminars in Oncology Nursing, 31(4), 338-347. https:// doi.org/10.1016/j.soncn.2015.08.009

Flynn, K. E., Reese, J. B., Jeffery, D. D., Abernethy, A. P., Lin, L., Shelby, R. A.,...Weinfurt, K. P. (2012). Patient experiences with communication about sex during and after treatment for cancer. Psychooncology, 21(6), 594-601. https:// doi.org/10.1002/pon.1947

Hill, E. K., Sandbo, S., Abramsohn, E., Makelarski, J., Wroblewski, K., Wenrich, E. R.,...Lindau, S. T. (2011). Assessing gynecologic and breast cancer survivors' sexual health care needs. Cancer, 117(12), 2643-2651. https:// doi.org/10.1002/cncr.25832

Huffman, L. B., Hartenbach, E. M., Carter, J., Rash, J. K., \& Kushner, D. M. (2016). Maintaining sexual health throughout gynecologic cancer survivorship: A comprehensive review and clinical guide. Gynecologic Oncology, 140(2), 359-368. https://doi.org/10.1016/j.ygyno.2015.11.010

Krychman, M. L., \& Katz, A. (2012). Breast cancer and sexuality: Multi-modal treatment options. Journal of Sexual Medicine, 9(1), 5-13; quiz 14-15. https://doi.org/10.1111/ j.1743-6109.2011.02566.x

Lindau, S. T., Abramsohn, E. M., \& Matthews, A. C. (2015). A manifesto on the preservation of sexual function in women and girls with cancer. American Journal of $\mathrm{Ob}$ stetrics and Gynecology, 213(2), 166-174. https://doi. org/10.1016/j.ajog.2015.03.039

Lindau, S. T., Abramsohn, E. M., Baron, S. R., Florendo, J., Haefner, H. K., Jhingran, A.,...Streicher, L. (2016). Physical 
examination of the female cancer patient with sexual concerns: What oncologists and patients should expect from consultation with a specialist. CA: A Cancer Journal for Clinicians, 66(3), 241-263. https://doi.org/10.3322/caac.21337

Miller, K. D., Nogueira, L., Mariotto, A. B., Rowland, J. H., Yabroff, R., Alfano, C. M.,...Siegel, R. L. (2019). Cancer treatment and survivorship statistics, 2019. CA: A Cancer Journal for Clinicians, 69(5), 363-385. https://doi. org/10.3322/caac. 21565

Morgan, B., \& Tarbi, E. (2016). The role of the advanced practice nurse in geriatric oncology care. Seminars in Oncology Nursing, 32(1), 33-43. https://doi.org/10.1016/j. soncn.2015.11.005

Park, E. R., Norris, R. L., \& Bober, S. L. (2009). Sexual health communication during cancer care: Barriers and recommendations. Cancer Journal, 15(1), 74-77. https://doi. org/10.1097/PPO.0b013e31819587dc

Rash, J. K., Lyle, K. D., Jairam-Thodla, A., \& Ioffe, Y. (2018). Delivery of gynecologic oncology care: Optimizing scope of advanced practice providers to enhance patient care: A Society of Gynecologic Oncology Position Paper. Gynecologic Oncology, 151(3), 494-500. https://doi.org/10.1016/j. ygyno.2018.09.018

Seaborne, L. A., Prince, R. J., \& Kushner, D. M. (2015). Sexual health education in U.S. physician assistant programs. Journal of Sexual Medicine, 12(5), 1158-1164. https://doi. org/10.1111/jsm.12879

Shulman, L. N. (2013). Efficient and effective models for integrating advanced practice professionals into oncology practice. American Society of Clinical Oncology Educational Book. https://doi.org/10.1200/EdBook AM.2013.33.e377

Yen, T. W. F., Laud, P. W., McGinley, E. L., Pezzin, L. E., \& Nattinger, A. B. (2019). Prevalence and scope of advanced practice provider oncology care among Medicare beneficiaries with breast cancer. Breast Cancer Research and Treatment, 179, 57-65. https://doi.org/10.1007/s10549019-05447-x

\section{Appendix A. Survey on WISH Program}

1. When you came to the WISH clinic, did you have a general understanding of issues related to sexual function after cancer?

2. When you came to the WISH clinic, did you have

a. Concerns about communication with your partner?

b. Vaginal dryness?

c. Pain with penetration?

d. Concerns about your sexual interest or libido?

e. Concerns about your ability to reach orgasm?

3. After your visits to the WISH clinic, did you experience improvement with your

a. General understanding of issues related to sexual function after cancer?

b. Concerns about communication with your partner?

c. Vaginal dryness?

d. Pain with penetration?

e. Concerns about your sexual interest or libido?

f. Concerns about your ability to reach orgasm?

4. After your visits to the WISH clinic, do you continue to have improvements with your a. General understanding of issues related to sexual function after cancer?

b. Concerns about communication with your partner?

c. Vaginal dryness?

d. Pain with penetration?

e. Concerns about your sexual interest or libido?

f. Concerns about your ability to reach orgasm?

5. Thinking about your experience overall, how helpful was your experience with the WISH clinic?

a. Not at all helpful

b. A little helpful

c. Somewhat helpful

d. Very helpful

e. Extremely helpful

6. After your experience with the WISH clinic

a. Has your overall sexual function improved?

b. Do you think your sexual functioning would be worse if you had NOT visited the WISH clinic?

c. Would you recommend the WISH clinic to other women with sexual problems after cancer? 